\title{
Biomechanical comparison of posterior intermediate screw fixation techniques with hybrid monoaxial and polyaxial pedicle screws in the treatment of thoracolumbar burst fracture: a finite element study
}

\author{
Huan Liu ${ }^{1 \dagger}$, Hongwei Wang ${ }^{2,3,4,5^{*}+}$ (D) Jun Liu², Changqing Li', Yue Zhou ${ }^{6}$ and Liangbi Xiang ${ }^{2}$
}

\begin{abstract}
Background: To compare the biomechanical characteristics of different posterior intermediate screw fixation techniques (ISFTs) with hybrid monoaxial pedicle screws (Mps) and polyaxial pedicle screws (Pps) used in thoracolumbar burst fractures.

Methods: Fixation techniques are compared with regard to the von Mises stress (VMS) of the instrumentations and intradiscal pressures (IDPs) of the adjacent segments by finite element method (FEM).

Results: The redistributed ROM of the fixation models with Pps fixed at the lowest segment was twice of the other fixation models in flexion and extension. The largest value of maximal VMS of a pedicle screw was located at the lowest pedicle screws when Mps are fixed at the lowest segment. The largest value of maximal VMS of the rods was decreased when more Pps are fixed at the models. Maximal IDPs of the upper adjacent segments were all larger than those of the lower adjacent segments. The maximal IDPs of the fixation model with MPs fixed at the lowest segment were larger than the other fixation models in flexion and extension.
\end{abstract}

Conclusions: Polyaxial pedicle screws could be placed at the upper or the median segment for the facilitated efficient application of the connecting rod. We should focus on the adjacent segmental degeneration especially the upper adjacent segment in the fixation model with Mps fixed at the lowest segment.

Keywords: Biomechanics, Thoracolumbar fracture, Hybrid, Monoaxial pedicle screw, Polyaxial pedicle screw

\section{Introduction}

Posterior short-segment pedicle screw fixation is widely used for the management of traumatic thoracolumbar burst fractures [1-3], posterior intermediate screw fixation technique (ISFT) at the fracture level can help improve and maintain the kyphosis correction, and the biomechanical stability also can be increased [4-18]. As a result, improved design and implantation techniques

\footnotetext{
* Correspondence: cplawhw@163.com

${ }^{\dagger}$ Huan Liu and Hongwei Wang contributed equally to this work.

${ }^{2}$ Department of Orthopedics, General Hospital of Northern Theater Command of Chinese PLA, Shenyang 110016, Liaoning, China

${ }^{3}$ State Key Laboratory of Robotics, Shenyang Institute of Automation,

Chinese Academy of Science, Shenyang 110016, Liaoning, China

Full list of author information is available at the end of the article
}

of pedicle screws such as polyaxial pedicle screws have reduced the rate of a pedicle screw and rod breakage and facilitated efficient application of the connecting rod without undue stress on the construct $[5,11,12,19-21]$. If the heads of the pedicle screws are not in a straight line, polyaxial pedicle screws should be placed for the facilitated efficient application of the connecting rod. Compared to a monoaxial screw design, the compression and bending strength at the polyaxial head was reduced because of its own specific structural design [20,21], but no studies have compared the hybrid monoaxial pedicle screw (Mps) and polyaxial pedicle screw (Pps) fixation techniques with regard to the range of motion (ROM),

(c) The Author(s). 2019 Open Access This article is distributed under the terms of the Creative Commons Attribution 4.0 International License (http://creativecommons.org/licenses/by/4.0/), which permits unrestricted use, distribution, and reproduction in any medium, provided you give appropriate credit to the original author(s) and the source, provide a link to the Creative Commons license, and indicate if changes were made. The Creative Commons Public Domain Dedication waiver (http://creativecommons.org/publicdomain/zero/1.0/) applies to the data made available in this article, unless otherwise stated. 
von Mises stress (VMS) of the instrumentations, and intradiscal pressures (IDPs) of the adjacent segments.

The focus of our research is to find how to provide sufficient biomechanical stability with hybrid Mps and Pps (how many Pps and location of the Pps to put) on the premise of ensuring convenient placement of rods. In the current study, biomechanical characteristics of fixation techniques including MMM (6 Mps fixated at three levels), PPP (6 Pps fixated at three levels), PMM (2 Pps fixated at upper level, 4 Mps fixated at lower two levels), MPM (4 Mps fixated at upper and lower two levels, 2 Pps fixated at median level), MMP(4 Mps fixated at upper two levels, 2 Pps fixated at lower level), $\operatorname{MPP}(2$ Mps fixated at upper levels, 4 Pps fixated at lower two levels), PMP (2 Mps fixated at median level, 4 Pps fixated at upper and lower levels), and PPM (4 Pps fixated at upper two levels, 2 Mps fixated at lower level) were compared using finite element methods, redistributed ROM, VMS of instrumentations, and IDPs of the adjacent segment under displacement loading which was evaluated.

\section{Materials and methods}

Finite element model (FEM) and assessment indexes

A finite element model including 7 vertebrae and 6 discs between T9 and L3 of the spine obtained from 64 spiral computed tomography (CT) images of a 40-year-old healthy male $(65 \mathrm{~kg}$ and $175 \mathrm{~cm})$ without a history of spinal injury, osteoporosis, and radiographic evidence of degeneration was reconstructed and analyzed using finite element analysis software $[6,22,23]$. The CT images were scanned and imported into Mimics 10.0 (Materialise, Belgium). The surface model was then exported into Rapidform 2006 (INUS, Korea) to generate and enhance the quality of the solid model. Eventually, the model was imported into Abaqus 6.9 (Simulia) for meshing. Each vertebral body consisted of cortical bone and cancellous bone, and each vertebral disc was composed of nucleus pulposus, annulus fibrosus, and endplates. Posterior elements were built separately from the vertebral bodies. Based on a Boolean operation, the lower half of the T12 segment was resected, and the structure of the posterior part was reserved to establish a finite element model of an unstable thoracolumbar fracture. Surface-to-surface contact was defined between articulation facets. We have built the intact normal spine model and fractured spine model. The intact spine model without implants had a total of 20,924 nodes and 72,055 elements which including 48,099 tetrahedron elements, 5212 hexahedral elements, 1236 spar elements, and 17,508 shell elements (Fig. 1). We have used a truss element to replace the ligament, and the thickness of the shell element was $0.4 \mathrm{~mm}$.

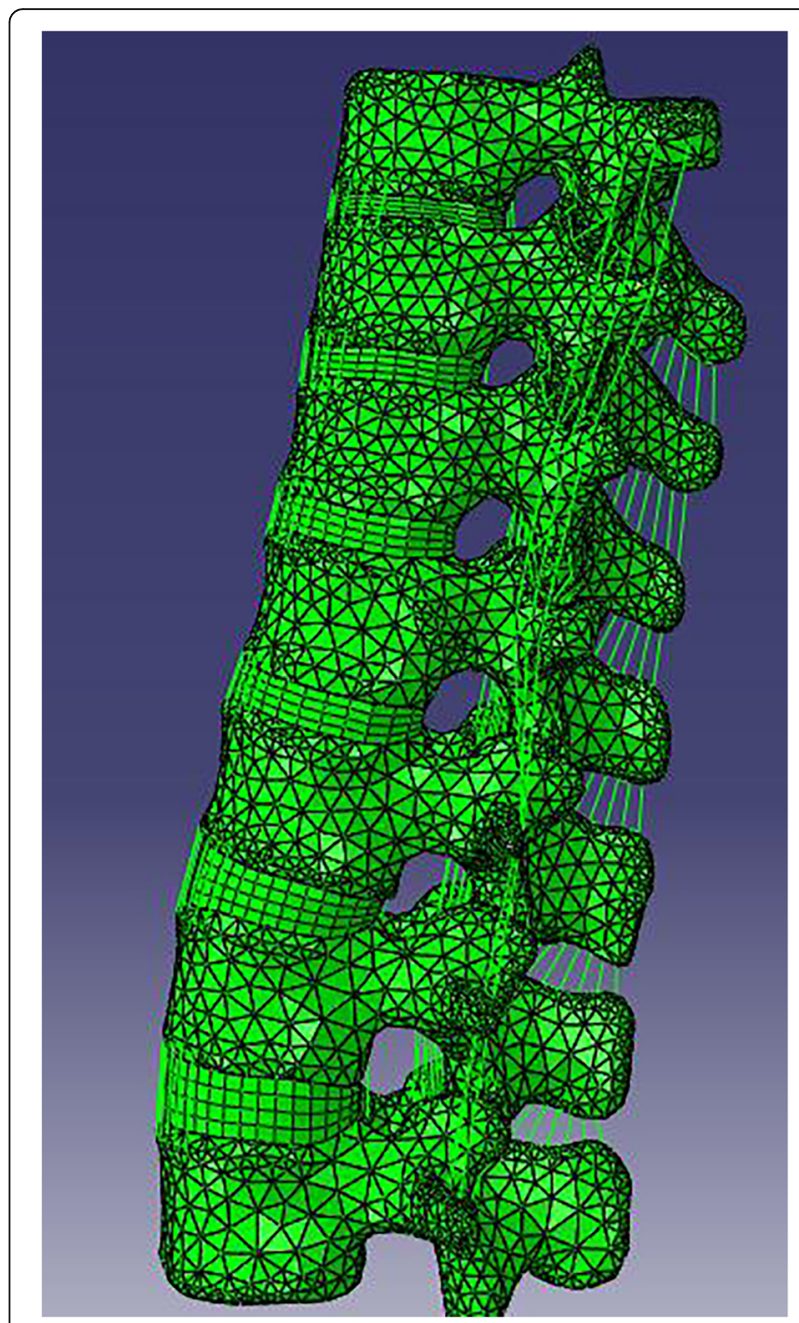

Fig. 1 Finite element model: intact spine model. The intact spine model had a total of 20,924 nodes and 72,055 elements

This was a prospective study to assess the biomechanical characteristics of different posterior intermediate screw fixation techniques with hybrid Mps and Pps used in thoracolumbar burst fracture model. Fixation models were described as MMM, PPP, PMM, MPM, MMP, MPP, PMP, and PPM (Figs. 2 and 3) which may be used in the clinical practice. Surface-to-surface contact was defined between articulation facets. The element types, material properties, ligamentary cross-sectional area, and implants are shown in our previous study [6].

The screw diameter was $6 \mathrm{~mm}$, and the screw length was $45 \mathrm{~mm}$. The pedicle screws in the current study included Mps and Pps. The constraint was defined between polyaxial pedicle screw heads and shafts. However, a load limitation was defined. Surface-to-surface contact was defined between polyaxial pedicle screw heads and shafts. The screw tilt (the maximal deviation of the long axis of the screw away from perpendicular to the longitudinal rod) was $25^{\circ}$, the static torque was $8 \mathrm{Nm}$ which meant that 

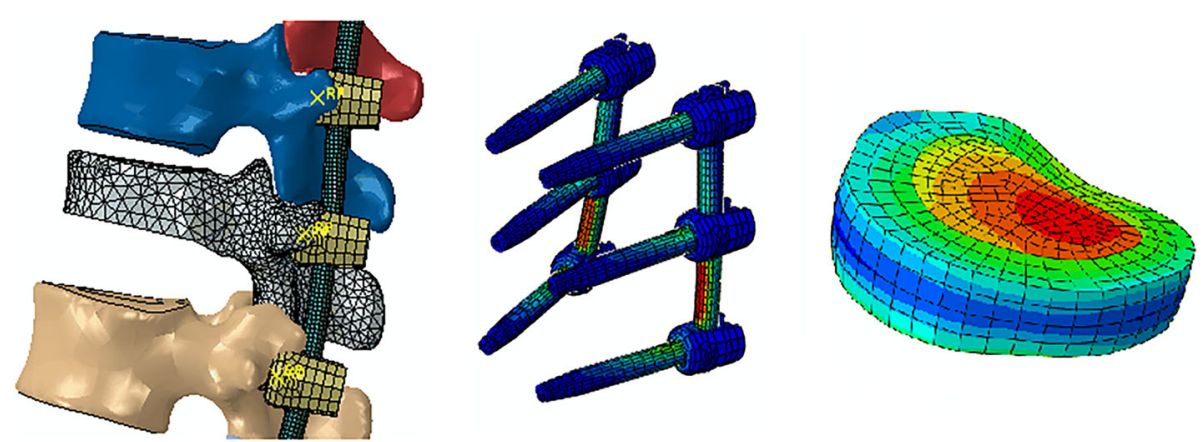

Fig. 2 Finite element models: fracture and fixation model. Graphical figures showing the von Mises stress of pedicle screw and disc models

the polyaxial pedicle screw heads will move relative to the shafts when the torque between the heads and shafts reached $8 \mathrm{Nm}$. These parameters are referred to as the polyaxial pedicle screw of Sofamor. The top surface of T9 was applied by a pure moment of $10 \mathrm{Nm}$ combined with a pre-compressive load of $150 \mathrm{~N}$, the inferior endplate of L3 was constrained in all degrees of freedom (Fig. 4). To validate the rationalities of the models, including model simplification, material properties, boundary conditions, and loads, a moment of $10 \mathrm{Nm}$ and a compressive load of 150 $\mathrm{N}$ were applied to the reference point. The range of motion (ROM) among different models was compared in our previous study [6]. There is little difference between the models. Therefore, the models in the present study are effective for further analyses.

We measured the ROM of the intact spine model T9L3 under flexion, extension, left/right lateral bending, and left/right axial rotation and then applied ROM displacement loading to the four fixation models. The redistributed ROM of the T11-L1 segment, the largest maximal VMS of the pedicle screws and rods, and IDPs of the adjacent segment under displacement loading were evaluated. The procedure was approved by the ethics committee of Xinqiao Hospital, and the patients provided written informed consent to participate in this study.



Fig. 3 Experiment grouping design 


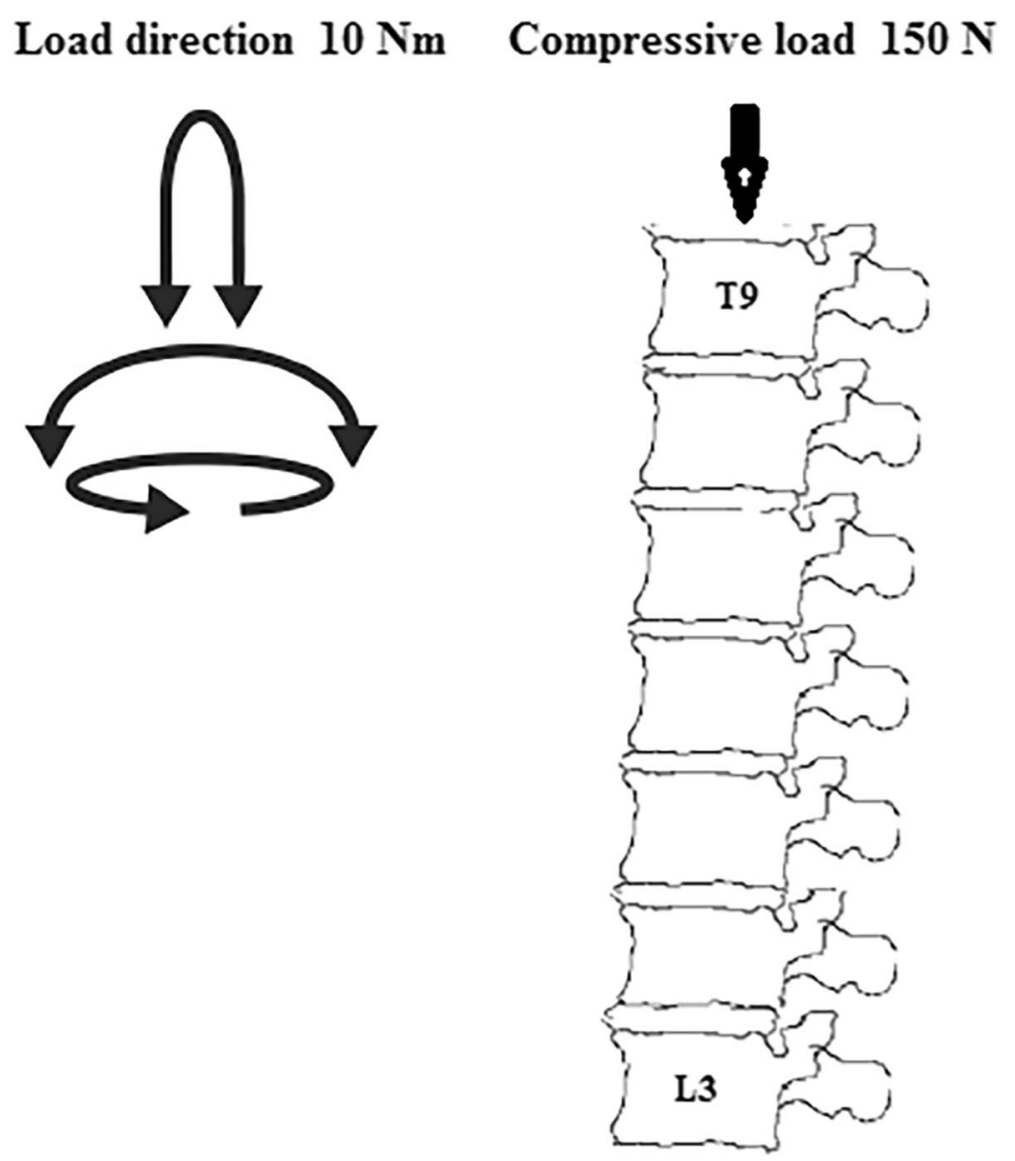

Fig. 4 Schematic figure to show the model, boundary conditions, and applied loads

\section{Statistical analysis}

We used SPSS 15.0 software (SPSS Inc., Illinois, USA) to perform all statistical analyses, and $P<0.05$ was considered significant (two-tailed). The independent sample $t$ test was used to compare the means.

\section{Results}

\section{ROM of the FEMs}

The fixation models presented with a decreased ROM than the intact normal spine model (Table 1). The redistributed
ROM of the MMM model in flexion, extension, and axial rotation was the smallest. The redistributed ROM of the fixation models with Pps fixed at the lowest segment was twice of the other fixation models in flexion and extension (Fig. 5). There were significant differences between the fixation models with Pps fixed at the lowest segment or not in the flexion $\left(8.0 \pm 0.1^{\circ}, 3.5 \pm 0.9^{\circ}, P=0.002\right)$ and extension $\left(6.7 \pm 0.1^{\circ}, 3.1 \pm 0.8^{\circ}, P=0.003\right)$, no significant differences in the axial rotation $\left(4.7 \pm 0.7^{\circ}, 3.1 \pm 1.3^{\circ}, P=0.073\right)$ and lateral bending $\left(3.3 \pm 0.3^{\circ}, 2.6 \pm 0.5^{\circ}, P=0.058\right)$.

Table 1 The ROM of the FEMs $\left(^{\circ}\right)$

\begin{tabular}{|c|c|c|c|c|c|c|c|c|c|}
\hline Variable & Normal & MMM & PPP & PMM & MPM & MMP & MPP & PMP & PPM \\
\hline Flexion & 8.3 & 2.5 & 8.1 & 3.8 & 3.1 & 7.9 & 8.2 & 8.0 & 4.7 \\
\hline Extension & 10.0 & 2.1 & 6.7 & 3.5 & 2.7 & 6.7 & 6.7 & 6.8 & 3.9 \\
\hline Axial rotation & 6.3 & 1.7 & 5.2 & 3.2 & 2.7 & 3.7 & 5.0 & 4.8 & 4.8 \\
\hline Lateral bending & 8.5 & 2.0 & 3.0 & 3.1 & 2.9 & 3.1 & 3.3 & 3.7 & 2.3 \\
\hline
\end{tabular}

MMM 6 Mps fixated at three levels; PPP 6 Pps fixated at three levels; PMM 2 Pps fixated at upper level, 4 Mps fixated at lower two levels; MPM 4 Mps fixated at upper and lower two levels, 2 Pps fixated at median level; MMP 4 Mps fixated at upper two levels, 2 Pps fixated at lower level; MPP 2 Mps fixated at upper levels, 4 Pps fixated at lower two levels; PMP 2 Mps fixated at median level, 4 Pps fixated at upper and lower levels; PPM 4 Pps fixated at upper two levels, 2 Mps fixated at lower level 


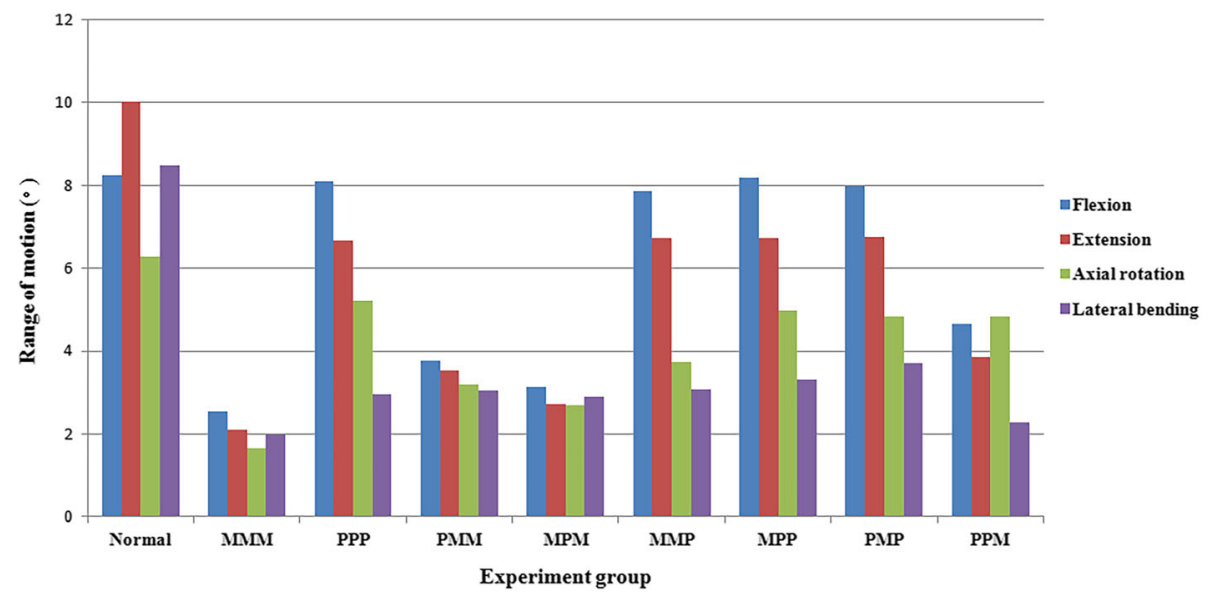

Fig. 5 ROM of different experiment groups under different states of motion

\section{VMS of the pedicle screws and rods}

The largest and smallest value of maximal VMS of a pedicle screw was $382.6 \mathrm{MPa}$ in the PMP model and 136.9 MPa in the PPP model, respectively (Table 2). The largest value of maximal VMS of a pedicle screw was located at the lowest pedicle screws when Mps are fixed at the lowest segment. The largest and smallest value of maximal VMS of the rod was $439.9 \mathrm{MPa}$ in the MMM model and $341.7 \mathrm{MPa}$ in the PPP model, respectively. The largest value of maximal VMS of the rods was decreased when more Pps are fixed at the models (Table 2), but there were no significant differences between the fixation models with two Pps fixed and models with four Pps fixed $(429.2 \pm 10.3,409.8 \pm$ 15.5, $P=0.145)$.

\section{IDPs of the adjacent segments}

Maximal IDPs of the adjacent segment was observed in the lateral bending. Maximal IDPs of the upper adjacent segments were all larger than those of the lower adjacent segments (Table 3). The maximal IDPs of the fixation model with Mps fixed at the lowest segment were larger than the other models in flexion and extension (Fig. 6). With regard to the upper adjacent segments, there were significant differences between the fixation models with
Mps fixed at the lowest segment in the flexion (1.9 \pm $0.1,1.3 \pm 0.1, P=0.000)$ and extension $(2.2 \pm 0.1,1.8 \pm$ $0.1, P=0.001)$, no significant differences in the axial rotation $(1.3 \pm 0.2,1.2 \pm 0.1, P=0.235)$ and lateral bending $(2.5 \pm 0.3,2.4 \pm 0.3, P=0.902)$. With regard to the lower adjacent segments, there were significant differences between the fixation models with Mps fixed at the lowest segment or not in the flexion $(0.7 \pm 0.1,0.4 \pm 0.1$, $P=0.000)$ and extension $(1.0 \pm 0.2,0.6 \pm 0.1, P=0.017)$, no significant differences in the axial rotation $(0.8 \pm 0.1$, $0.9 \pm 0.2, P=0.072)$ and lateral bending $(1.5 \pm 0.1,1.5 \pm$ $0.1, P=1.000)$.

\section{Discussion}

Posterior intermediate screw fixation at the fracture level can help improve and maintain the kyphosis correction, and the biomechanical stability also can be increased [4-18]. However, no studies have compared the hybrid Mps and Pps fixation techniques with regard to the ROM, VMS of the instrumentations, and IDPs of the adjacent segments. Our previous study suggested that the intermediate screw fixation technique can significantly increase the stability of the spine in both the Mps fixation group and Pps fixation group. However, the Mps fixation group exhibited more stability in flexion and

Table 2 Value, location, and state of motion of pedicle screws/value and state of motion of rods with regard to the LVMS

\begin{tabular}{llllllllll}
\hline Variable & & MMM & PPP & PMM & MPM & MMP & MPP & PMP & PPM \\
\hline Pedicle screws & Value (MPa) & 332.8 & 136.9 & 328.2 & 321.3 & 313.9 & 266.1 & 382.6 & 363.1 \\
& Location & Lower & Upper & Lower & Lower & Median & Upper & Median & Lower \\
& State of motion & Flexion & Flexion & Flexion & Flexion & Rotation & Rotation & Rotation & Rotation \\
\multirow{2}{*}{ Rods } & Value (MPa) & 439.9 & 341.7 & 439 & 430.3 & 418.4 & 414.1 & 392.6 & 422.7 \\
& State of motion & Flexion & Rotation & Flexion & Flexion & Rotation & Rotation & Rotation & Flexion
\end{tabular}

MMM 6 Mps fixated at three levels; PPP 6 Pps fixated at three levels; PMM 2 Pps fixated at upper level, 4 Mps fixated at lower two levels; MPM 4 Mps fixated at upper and lower two levels, 2 Pps fixated at median level; MMP 4 Mps fixated at upper two levels, 2 Pps fixated at lower level; MPP 2 Mps fixated at upper levels, 4 Pps fixated at lower two levels; PMP 2 Mps fixated at median level, 4 Pps fixated at upper and lower levels; PPM 4 Pps fixated at upper two levels, 2 Mps fixated at lower level 
Table 3 The IDPs of the upper and lower adjacent segments (MPa)

\begin{tabular}{|c|c|c|c|c|c|c|c|c|c|c|c|c|c|c|c|c|c|c|}
\hline \multirow[t]{2}{*}{ Variable } & \multicolumn{2}{|c|}{ Normal } & \multicolumn{2}{|c|}{ MMM } & \multicolumn{2}{|l|}{ PPP } & \multicolumn{2}{|c|}{ PMM } & \multicolumn{2}{|c|}{ MPM } & \multicolumn{2}{|c|}{ MMP } & \multicolumn{2}{|c|}{ MPP } & \multicolumn{2}{|c|}{ PMP } & \multicolumn{2}{|c|}{ PPM } \\
\hline & $\mathrm{U}$ & L & $\mathrm{U}$ & L & $U$ & L & $U$ & L & U & L & U & L & U & L & $U$ & L & $\mathrm{U}$ & L \\
\hline Flexion & 1.4 & 0.6 & 2.1 & 0.7 & 1.4 & 0.3 & 1.9 & 0.7 & 1.8 & 0.7 & 1.3 & 0.4 & 1.3 & 0.4 & 1.3 & 0.4 & 1.8 & 0.8 \\
\hline Extension & 1.4 & 0.8 & 2.3 & 0.9 & 1.6 & 0.5 & 2.1 & 0.8 & 2.2 & 0.9 & 1.8 & 0.7 & 1.8 & 0.6 & 1.8 & 0.7 & 2.1 & 1.2 \\
\hline Axial rotation & 0.9 & 0.6 & 1.5 & 0.8 & 1.2 & 0.7 & 1.3 & 0.7 & 1.4 & 0.7 & 1.3 & 1.0 & 1.1 & 1.0 & 1.2 & 1.0 & 1.1 & 0.8 \\
\hline Lateral bending & 2.0 & 1.1 & 2.9 & 1.5 & 2.8 & 1.5 & 2.3 & 1.4 & 2.3 & 1.5 & 2.3 & 1.4 & 2.3 & 1.6 & 2.3 & 1.6 & 2.3 & 1.7 \\
\hline
\end{tabular}

MMM 6 Mps fixated at three levels; PPP 6 Pps fixated at three levels; PMM 2 Pps fixated at upper level, 4 Mps fixated at lower two levels; MPM 4 Mps fixated at upper and lower two levels, 2 Pps fixated at median level; MMP 4 Mps fixated at upper two levels, 2 Pps fixated at lower level; MPP 2 Mps fixated at upper levels, 4 Pps fixated at lower two levels; PMP 2 Mps fixated at median level, 4 Pps fixated at upper and lower levels; PPM 4 Pps fixated at upper two levels, 2 Mps fixated at lower level

extension than the Pps fixation group [5]. Fixation models including MMM, PPP, PMM, MPM, MMP, MPP, PMP, and PPM showed less ROM than the intact normal spine model, and the redistributed ROM of the MMM model in flexion, extension, and axial rotation was the smallest. The redistributed ROM of the fixation models with Pps fixed at the lowest segment was twice of the other fixation models in flexion and extension. The redistributed ROMs of the PMM and MPM models were very close to the MMM model. The phenomenon can be explained for that polyaxial pedicle screw heads are vulnerable to fatigue failure; the region between the screw head and shaft was found to fail first in many biomechanical studies $[19,20,24]$. Through the study, we can see that if the heads of the pedicle screws are not in a straight line, we should place the polyaxial pedicle screws at the upper or the median segment.

The largest and smallest value of maximal VMS of a pedicle screw was $382.6 \mathrm{MPa}$ in the PMP model and 136.9 $\mathrm{MPa}$ in the PPP model, respectively. The largest value of maximal VMS of a pedicle screw was located at the lowest pedicle screws when Mps are fixed at the
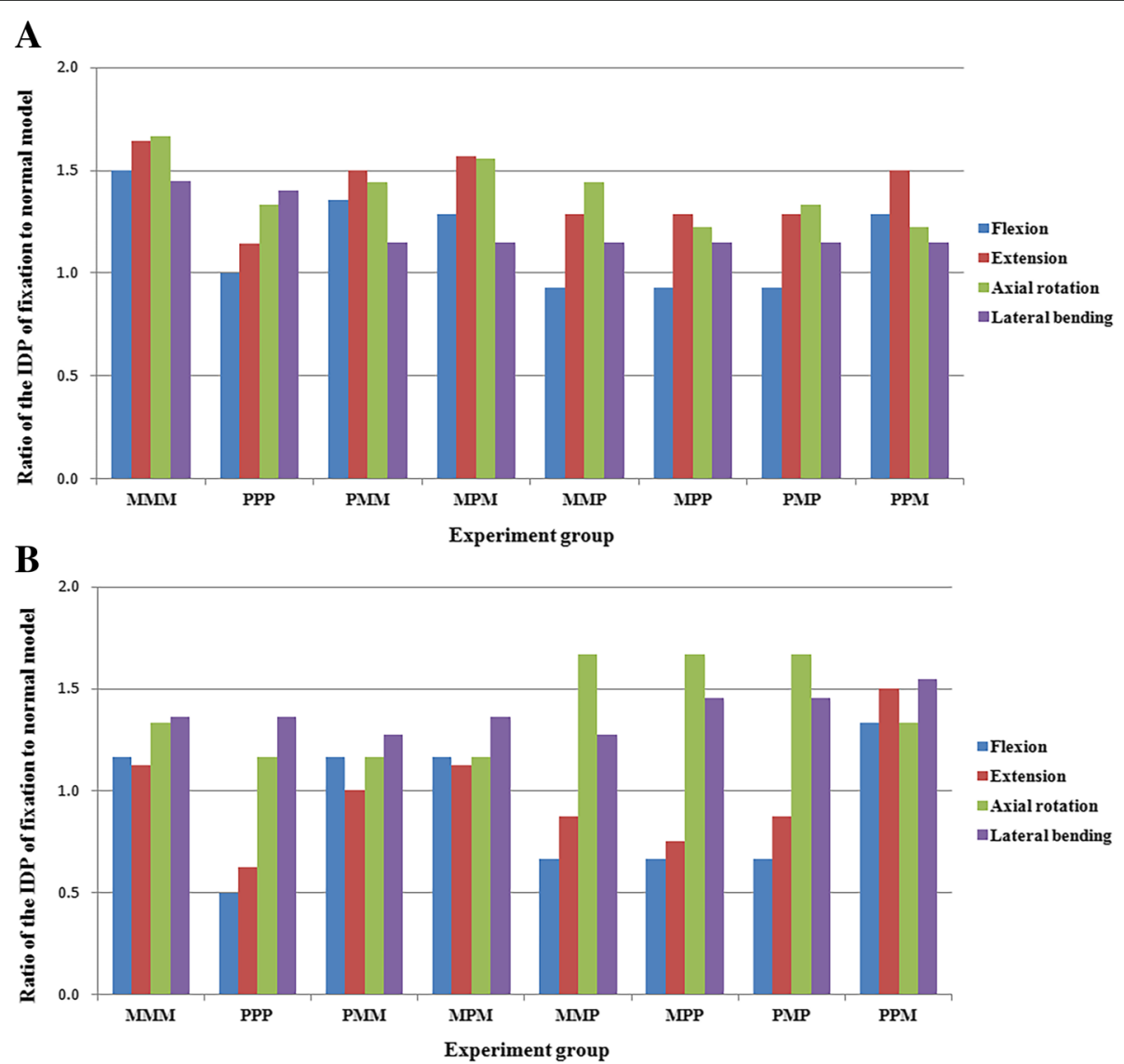

Fig. 6 Ratio of the adjacent segmental IDPs of the fixation model to the normal model. a Ratio of the upper adjacent segmental IDPs of the fixation model to the normal model. $\mathbf{b}$ Ratio of the lower adjacent segmental IDPs of the fixation model to the normal model 
lowest segment. These results may suggest that the PMP technique can increase the VMS of the pedicle screws. Upon suspecting that a pedicle screw is broken, we must focus on the median pedicle screws in PMP technique and the lower pedicle screws when Mps are fixed at the lowest segment. The largest and smallest value of maximal VMS of the rod was $439.9 \mathrm{MPa}$ in the MMM model and 341.7 $\mathrm{MPa}$ in the PPP model, respectively. The largest value of maximal VMS of the rods was decreased when more Pps are fixed at the models. These results may suggest that the Pps technique can decrease the VMS of the rods. Upon suspecting that a rod is broken, we must focus on the MMM, PMM, and MPM fixation techniques.

In our study, the maximal IDPs of the adjacent segment were observed in the lateral bending. Maximal IDPs of the upper adjacent segments were all larger than those of the lower adjacent segments in the fixation models. These results were consistent with previous studies [25-28], the upper ASD can develop more easily than the lower ASD after the fusion surgery. The maximal IDPs of the adjacent segment in the fixation model with Mps fixed at the lowest segment were larger than the other fixation models in flexion and extension and larger than the normal model in all states of motion. These results were consistent with previous studies that noted that fusion accelerates degenerative changes at the adjacent level compared with natural history [29-31].

This study has several limitations. It is necessary to discuss several factors, including different persons, muscle force, ribs, and length and diameter of pedicle screws, for a more clinically feasible conclusion because these factors can influence finite element analysis results.

\section{Conclusion}

ROM of the fixation models with Pps fixed at the lowest segment was twice of the other fixation models in flexion and extension, and the largest value of maximal VMS of the rods was decreased when more Pps are fixed at the models. The largest value of maximal VMS of a pedicle screw was located at the lowest pedicle screws, and the maximal adjacent segmental IDPs of the fixation model were larger than the other models in flexion and extension when Mps are fixed at the lowest segment. Through the study, we can see that if the heads of the pedicle screws are not in a straight line, the polyaxial pedicle screws should be placed at the upper or the median segment for the facilitated efficient application of the connecting rod. Upon suspecting instrumentation failure, we must focus on the median pedicle screws in the PMP fixation technique and the lower pedicle screws when Mps are fixed at the lowest segment and the rod in the MMM, PMM, and MPM fixation techniques. We should focus on the adjacent segmental degeneration especially the upper adjacent segment in the fixation model with Mps fixed at the lowest segment.

\section{Abbreviations}

IDPs: Intradiscal pressures; ISFT: Intermediate screw fixation technique; MMM: 6 Mps fixated at three levels; MMP: 4 Mps fixated at upper two levels, 2 Pps fixated at lower level; MPM: 4 Mps fixated at upper and lower two levels, 2 Pps fixated at median level; MPP: 2 Mps fixated at upper levels, 4 Pps fixated at lower two levels; Mps: Monoaxial pedicle screws; PMM: 2 Pps fixated at upper level, 4 Mps fixated at lower two levels; PMP: 2 Mps fixated at median level, 4 Pps fixated at upper and lower levels; PPM: 4 Pps fixated at upper two levels, 2 Mps fixated at lower level; PPP: 6 Pps fixated at three levels; Pps: Polyaxial pedicle screws; ROM: Range of motion; VMS: von Mises stress

\section{Acknowledgements}

The authors would like to thank Hua Yang, Zhongjun Mo, Yirong Zhao, and Guofei Zeng for his assistance with the data analysis and manuscript revision. This work was supported by the Foundation of the Liaoning Province Doctor Startup Fund (201601389), the State Key Laboratory of Robotics (2017-001), the Open Project Program of the State Key Lab of CAD\&CG (A1718), the Open Project Program of the State Key Laboratory of Trauma, Burn and Combined Injury (SKLKF201705), the State Key Laboratory of Materials Processing and Die \& Mould Technology (P2018-011), and the National Natural Science Foundation of China (11602063).

\section{Funding}

This work was supported by the Foundation of the Sichuan Province Applied Basic Research (2018JY0402), the Liaoning Province Doctor Startup Fund (201601389), the State Key Laboratory of Robotics (2017-001), the Open Project Program of the State Key Lab of CAD\&CG (A1718), the Open Project Program of the State Key Laboratory of Trauma, Burn and Combined Injury (SKLKF201705), the State Key Laboratory of Materials Processing and Die \& Mould Technology (P2018- 011) and the National Natural Science Foundation of China (11602063).

\section{Availability of data and materials}

The datasets used and analyzed during the current study are available from the corresponding author upon reasonable request.

\section{Authors' contributions}

$H W, H L, C L$, and $L X$ designed and participated in the whole process of the study and drafted the manuscript. All authors read and approved the final manuscript.

\section{Ethics approval and consent to participate}

The procedure was approved by the ethics committee of Xinqiao Hospital, and the patients provided written informed consent to participate in this study.

\section{Consent for publication}

My manuscript contains an individual person's data, consent to publish has been obtained from that person.

\section{Competing interests}

The authors declare that they have no competing interests.

\section{Publisher's Note}

Springer Nature remains neutral with regard to jurisdictional claims in published maps and institutional affiliations.

\section{Author details}

'Department of Orthopedics, Affiliated Traditional Chinese Medicine Hospital, Southwest Medical University, Luzhou 646000, China. ${ }^{2}$ Department of Orthopedics, General Hospital of Northern Theater Command of Chinese PLA, Shenyang 110016, Liaoning, China. ${ }^{3}$ State Key Laboratory of Robotics, Shenyang Institute of Automation, Chinese Academy of Science, Shenyang 110016, Liaoning, China. ${ }^{4}$ State Key Laboratory of Materials Processing and Die \& Mould Technology, Huazhong University of Science and Technology, Wuhan 430074, Hubei, China. ${ }^{5}$ State Key Laboratory of Trauma, Burn and Combined Injury, The Third Military Medical University, Chongqing 400038, China. ${ }^{6}$ Department of Orthopedics, Xinqiao Hospital, The Third Military Medical University, Chongqing 400037, China. 
Received: 1 August 2018 Accepted: 10 April 2019

Published online: 08 May 2019

\section{References}

1. Verlaan JJ, Diekerhof CH, Buskens E, van der Tweel I, Verbout AJ, Dhert WJ, et al. Surgical treatment of traumatic fractures of the thoracic and lumbar spine: a systematic review of the literature on techniques, complications, and outcome. Spine (Phila Pa 1976). 2004;29(7):803-14.

2. Shen WJ, Liu TJ, Shen YS. Nonoperative treatment versus posterior fixation for thoracolumbar junction burst fractures without neurologic deficit. Spine (Phila Pa 1976). 2001;26(9):1038-45.

3. Wang H, Zhang Y, Xiang Q, Wang X, Li C, Xiong H, et al. Epidemiology of traumatic spinal fractures: experience from medical university-affiliated hospitals in Chongqing, China, 2001-2010. J Neurosurg Spine. 2012;17(5): 459-68.

4. Baaj AA, Reyes PM, Yaqoobi AS, Uribe JS, Vale FL, Theodore N, et al. Biomechanical advantage of the index-level pedicle screw in unstable thoracolumbar junction fractures. J Neurosurg Spine. 2011;14(2):192-7.

5. Wang H, Li C, Liu T, Zhao WD, Zhou Y. Biomechanical efficacy of monoaxial or polyaxial pedicle screw and additional screw insertion at the level of fracture, in lumbar burst fracture: an experimental study. Indian J Orthop. 2012;46(4):395-401.

6. Li C, Zhou Y, Wang H, Liu J, Xiang L. Treatment of unstable thoracolumbar fractures through short segment pedicle screw fixation techniques using pedicle fixation at the level of the fracture: a finite element analysis. PLoS One. 2014:9(6):e99156.

7. Kanna RM, Shetty AP, Rajasekaran S. Posterior fixation including the fractured vertebra for severe unstable thoracolumbar fractures. Spine J. 2015;15(2):256-64.

8. Li K, Zhang W, Liu D, Xu H, Geng W, Luo D, et al. Pedicle screw fixation combined with intermediate screw at the fracture level for treatment of thoracolumbar fractures: a meta-analysis. Medicine (Baltimore). 2016;95(33): e4574.

9. Li K, Li Z, Ren X, Xu H, Zhang W, Luo D, et al. Effect of the percutaneous pedicle screw fixation at the fractured vertebra on the treatment of thoracolumbar fractures. Int Orthop. 2016;40(6):1103-10.

10. Sun C, Guan G, Liu X, Zhang H, Wang B. Comparison of short-segment pedicle fixation with versus without inclusion of the fracture level in the treatment of mild thoracolumbar burst fractures. Int J Surg. 2016;36(Pt A:352-7.

11. Wang H, Zhou Y, Li C, Liu J, Xiang L. Comparison of open versus percutaneous pedicle screw fixation using the sextant system in the treatment of traumatic thoracolumbar fractures. Clin Spine Surg. 2017;30(3): E239-46.

12. Wang H, Zhao Y, Mo Z, Han J, Chen Yu YH, et al. Comparison of short segment monoaxial and polyaxial pedicle screws fixation combined with intermediate screws in the traumatic thoracolumbar fractures: a finite element study and clinical radiographic review. Clinics. 2017;72(10):609-17.

13. Kose KC, Inanmaz ME, Isik C, Basar H, Caliskan I, Bal E. Short segment pedicle screw instrumentation with an index level screw and cantilevered hyperlordotic reduction in the treatment of type-A fractures of the thoracolumbar spine. Bone Joint J. 2014;96-B(4):541-7.

14. Norton RP, Milne EL, Kaimrajh DN, Eismont FJ, Latta LL, Williams SK. Biomechanical analysis of four- versus six-screw constructs for shortsegment pedicle screw and rod instrumentation of unstable thoracolumbar fractures. Spine J. 2014;14(8):1734-9.

15. Ökten Al, Gezercan Y, Özsoy KM, Ateş T, Menekşe G, Aslan A, et al. Results of treatment of unstable thoracolumbar burst fractures using pedicle instrumentation with and without fracture-level screws. Acta Neurochir (Wien). 2015;157(5):831-6.

16. Dobran $M$, Nasi $D$, Brunozzi $D$, di Somma $L$, Gladi M, lacoangeli $M$, et al. Treatment of unstable thoracolumbar junction fractures: short-segment pedicle fixation with inclusion of the fracture level versus long-segment instrumentation. Acta Neurochir (Wien). 2016;158(10):1883-9.

17. Ye C, Luo Z, Yu X, Liu H, Zhang B, Dai M. Comparing the efficacy of shortsegment pedicle screw instrumentation with and without intermediate screws for treating unstable thoracolumbar fractures. Medicine (Baltimore). 2017;96(34):e7893.

18. Ozdemir B, Kanat A, Erturk C, Batcik OE, Balik MS, Yazar U, et al. Restoration of anterior vertebral height by short-segment pedicle screw fixation with screwing of fractured vertebra for the treatment of unstable thoracolumbar fractures. World Neurosurg. 2017;99:409-17.
19. Fogel GR, Reitman CA, Liu W, Esses SI. Physical characteristics of polyaxialheaded pedicle screws and biomechanical comparison of load with their failure. Spine (Phila Pa 1976). 2003;28(5):470-3.

20. Stanford RE, Loefler AH, Stanford PM, Walsh WR. Multiaxial pedicle screw designs: static and dynamic mechanical testing. Spine (Phila Pa 1976). 2004; 29(4):367-75.

21. Shepard MF, Davies MR, Abayan A, Kabo JM, Wang JC. Effects of polyaxial pedicle screws on lumbar construct rigidity. J Spinal Disord Tech. 2002;15(3): 233-6.

22. Kim Y, Kim TW. Finite element analysis of the effects of a pedicle screw fixation nut loosening on lumbar interbody fusion based on the elastoplateau plasticity of bone characteristics. Spine (Phila Pa 1976). 2010;35(6): 599-606.

23. Kim HJ, Chun HJ, Moon SH, Kang KT, Kim HS, Park JO, et al. Analysis of biomechanical changes after removal of instrumentation in lumbar arthrodesis by finite element analysis. Med Biol Eng Comput. 2010;48(7): 703-9.

24. Kubosch D, Kubosch EJ, Gueorguiev B, Zderic I, Windolf M, Izadpanah K, et al. Biomechanical investigation of a minimally invasive posterior spine stabilization system in comparison to the Universal Spinal System (USS). BMC Musculoskelet Disord. 2016;17:134.

25. Rahm MD, Hall BB. Adjacent-segment degeneration after lumbar fusion with instrumentation: a retrospective study. J Spinal Disord. 1996;9(5):392-400.

26. Pellisé F, Hernández A, Vidal X, Minguell J, Martínez C, Villanueva C. Radiologic assessment of all unfused lumbar segments 7.5 years after instrumented posterior spinal fusion. Spine (Phila Pa 1976). 2007;32(5):574-9.

27. Chen CS, Cheng CK, Liu CL, Lo WH. Stress analysis of the disc adjacent to interbody fusion in lumbar spine. Med Eng Phys. 2001;23(7):483-91.

28. Nakashima H, Kawakami N, Tsuji T, Ohara T, Suzuki Y, Saito T, et al. Adjacent segment disease after posterior lumbar interbody fusion: based on cases with a minimum of 10 years of follow-up. Spine (Phila Pa 1976). 2015;40(14): E831-41.

29. Ekman P, Möller H, Shalabi A, Yu YX, Hedlund R. A prospective randomised study on the long-term effect of lumbar fusion on adjacent disc degeneration. Eur Spine J. 2009;18(8):1175-86.

30. Mannion AF, Leivseth G, Brox Jl, Fritzell P, Hägg O, Fairbank JC. ISSLS Prize winner: Long-term follow-up suggests spinal fusion is associated with increased adjacent segment disc degeneration but without influence on clinical outcome: results of a combined follow-up from 4 randomized controlled trials. Spine (Phila Pa 1976). 2014;39(17):1373-83.

31. Schulte TL, Leistra F, Bullmann V, Osada N, Vieth V, Marquardt B, et al. Disc height reduction in adjacent segments and clinical outcome 10 years after lumbar 360 degrees fusion. Eur Spine J. 2007;16(12):2152-8.

Ready to submit your research? Choose BMC and benefit from:

- fast, convenient online submission

- thorough peer review by experienced researchers in your field

- rapid publication on acceptance

- support for research data, including large and complex data types

- gold Open Access which fosters wider collaboration and increased citations

- maximum visibility for your research: over $100 \mathrm{M}$ website views per year

At BMC, research is always in progress.

Learn more biomedcentral.com/submissions 